\title{
Vaping safer than smoking but not without cardiovascular risk: A PET/MRI study
}

Mount

Sinai

TMII

\author{
A. Sahota1 ${ }^{1}$, S. Naidu11, A. Jacobi1 ${ }^{1}$, C. Giannarelli2, Z. Fayad1,2, V. Mani1. \\ 1 Icahn School of Medicine at Mount Sinai, Radiology- TMII, New York, USA. \\ 2Icahn School of Medicine at Mount Sinai, Medicine, New York, USA.
}

\section{Background and Aims:}

Electronic cigarettes (ECigs) are considered safer than smoking and promoted as a tool for smoking cessation. ECig cardiovascular safety however, is not well established. We examined cardiovascular consequences of ECig use by examining the entire atherosclerotic cascade (Figure 1) of young adults (18-30 years old) using noninvasive combined positron emission tomography $(\mathrm{PET}) /$ magnetic resonance imaging (MRI) and compared ECig users with age and gender matched smokers of traditional cigarettes and nonsmoking controls. We hypothesized that there will be differences in atherosclerotic disease as measured by PET/ MRI when comparing ECig users, traditional smokers and nonsmokers.

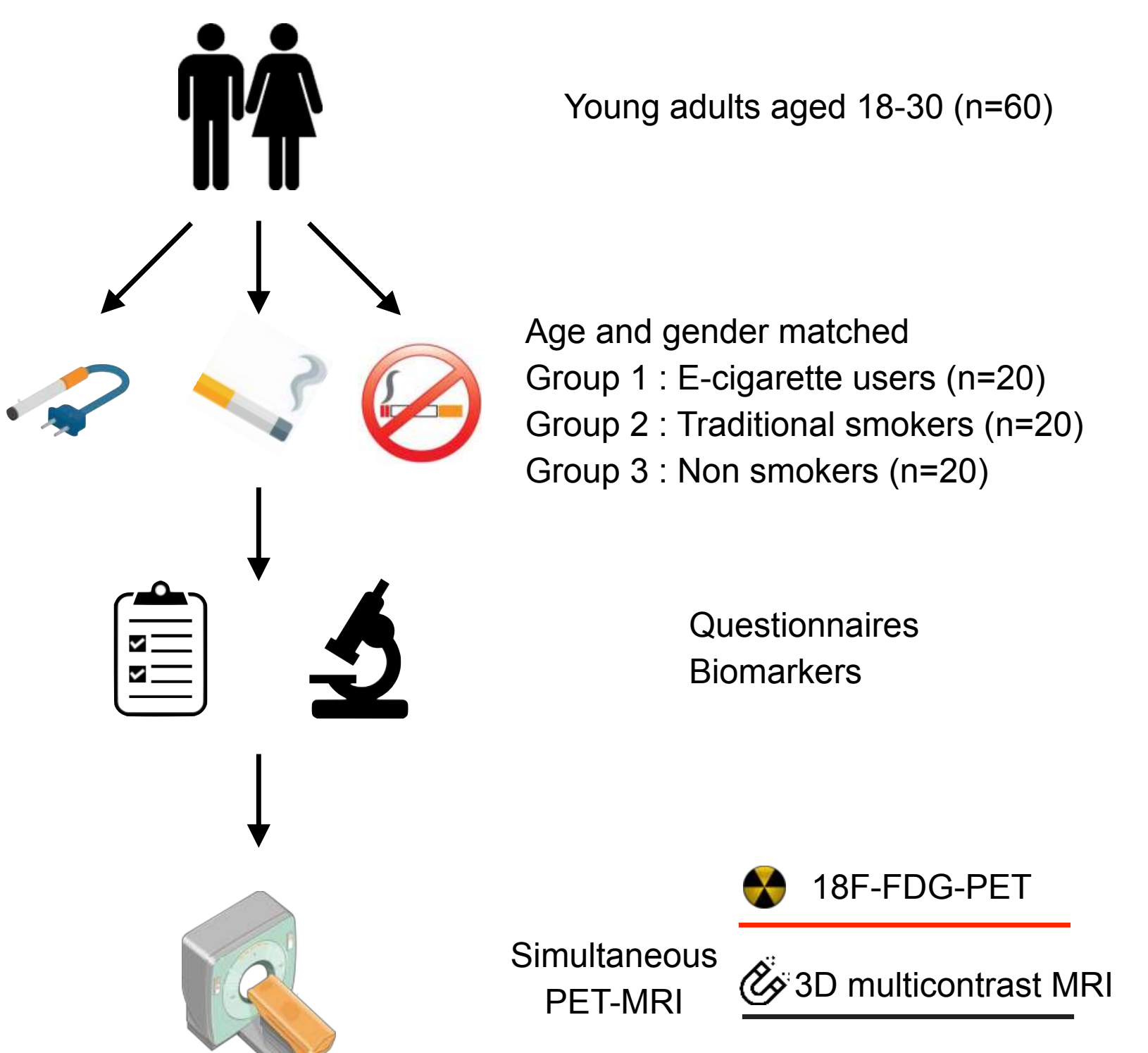

Figure 2: Study Design

\section{Methods:}

60 age and gender matched subjects were recruited (20 purely electronic cigarette users, 20 traditional smokers and 20 nonsmokers). Carotid PET/MRI was obtained to look at vascular inflammation(18-fluorodeoxyglucose(FDG)-PET) and plaque burden (multi-contrast MRI of vessel wall) in these individuals (Figure 2).

\section{Results:}

There were no differences in vascular inflammation as measured by 18-FDG-PET between ECig users, traditional smokers and nonsmokers. However, measures of plaque burden: wall area, normalized wall index, and wall thickness measured from MRI indicated that both smokers and ECig users were significantly higher compared to nonsmokers; with smokers having even higher values that Ecig users (Figure 3).

\section{Conclusions:}

Young adults ECig users, smokers and nonsmokers in our cohort did not exhibit differences in vascular inflammation; but smokers and EC users had significantly more plaque burden compared to matched nonsmokers. Ecig use may be safer than traditional cigarette use in terms of cardiovascular risk measured by PET/ MRI but these individuals have increased plaque burden compared to nonsmoking controls.

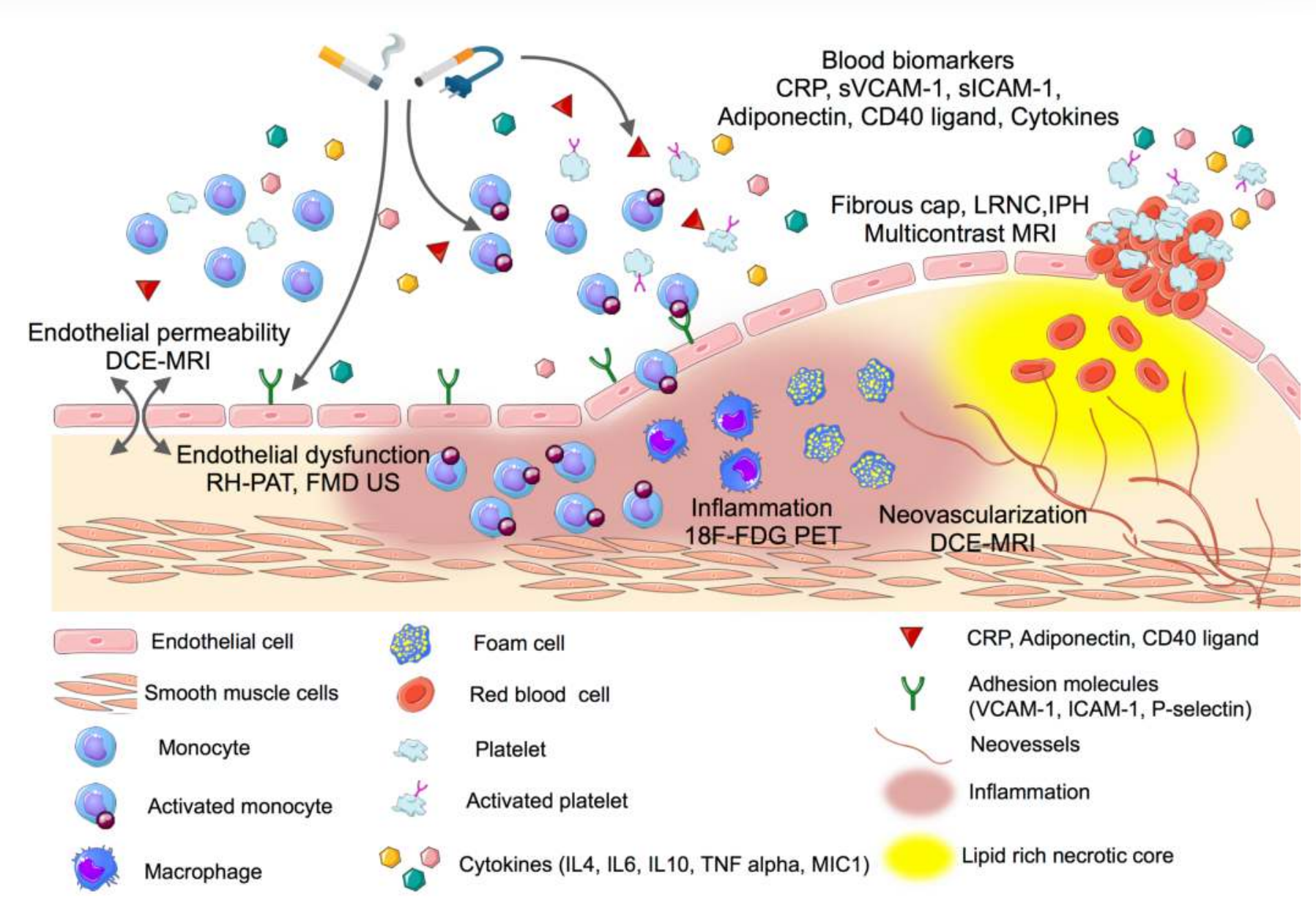

Figure 1: Role of cigarette smoke and/or e-cigarette vapor on the atherosclerotic disease progression and the imaging modalities that can be used to visualize them.

Table 1: Patient Demographics and Characteristics

\begin{tabular}{|l|c|c|c|c|}
\hline & $\begin{array}{c}\text { Cigarette } \\
\text { Smokers }\end{array}$ & $\begin{array}{c}\text { E-Cigarette } \\
\text { Vapers }\end{array}$ & Nonsmokers & p-value \\
\hline Age (yrs) & $27.0 \pm 3.05$ & $25.7 \pm 4.15$ & $24.6 \pm 1.94$ & 0.06 \\
\hline Height (in) & $68.2 \pm 3.4$ & $68.9 \pm 4.0$ & $67.6 \pm 3.7$ & 0.55 \\
\hline Weight (lbs) & $162 \pm 33$ & $177 \pm 32$ & $151 \pm 37$ & 0.02 \\
\hline BMI & $24.5 \pm 4.9$ & $26.1 \pm 3.0$ & $23.1 \pm 3.4$ & 0.03 \\
\hline Tot Chol (mg/dl) & $171 \pm 23$ & $177 \pm 34$ & $173 \pm 22$ & 0.72 \\
\hline TGC (mg/dl) & $117 \pm 127$ & $82 \pm 42$ & $80 \pm 40$ & 0.37 \\
\hline HDL (mg/dl) & $59 \pm 18$ & $56 \pm 14$ & $60 \pm 13$ & 0.64 \\
\hline LDL ( mg/dl) & $97 \pm 22$ & $99 \pm 21$ & $97 \pm 21$ & 0.95 \\
\hline Creatinine & $0.89 \pm 0.17$ & $0.88 \pm 0.14$ & $0.92 \pm 0.19$ & 0.69 \\
\hline Glucose (mg/dl) & $79 \pm 8.8$ & $81 \pm 12.3$ & $80 \pm 9.4$ & 0.91 \\
\hline
\end{tabular}
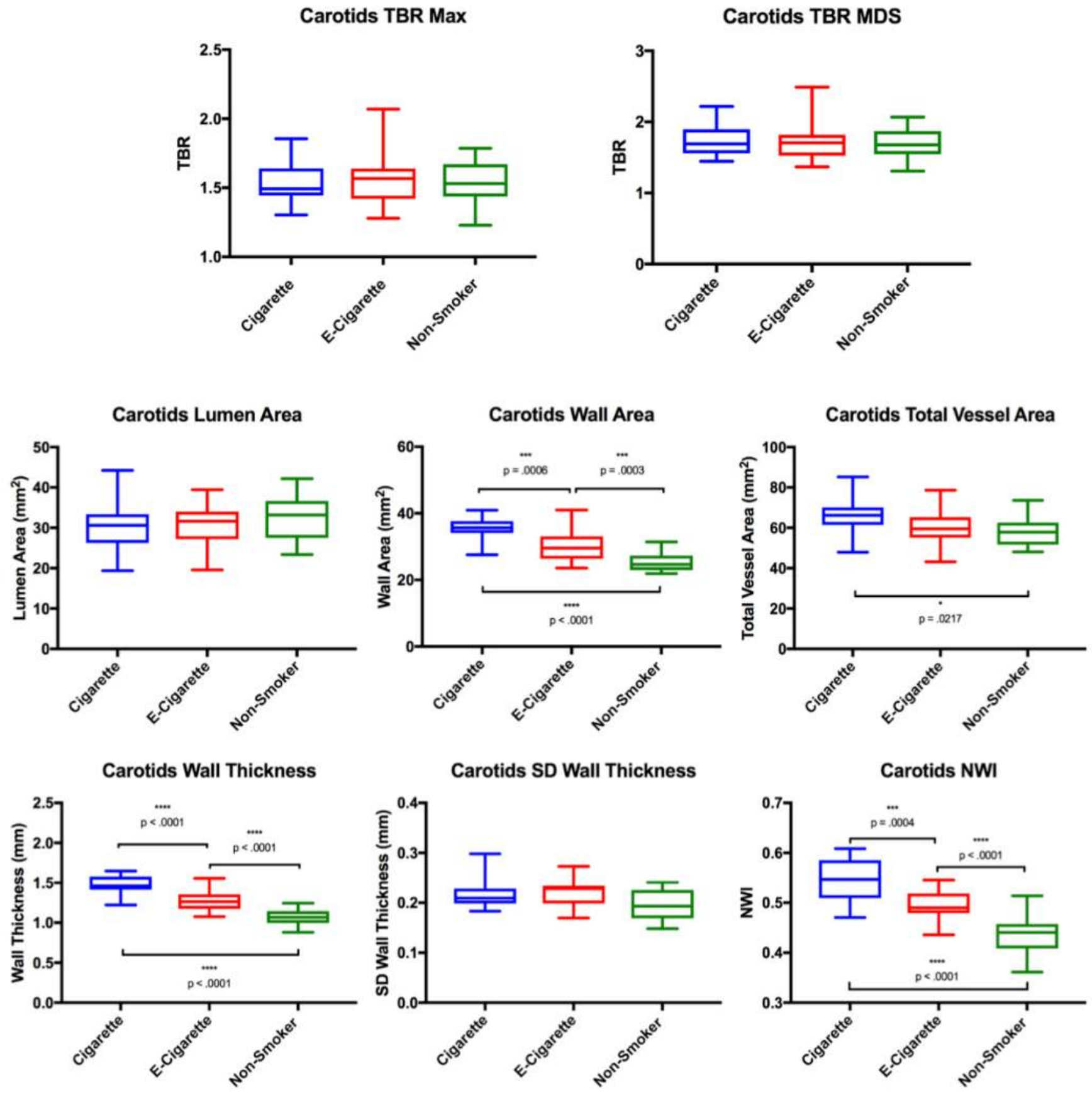

Figure 3: PET and MRI measures of atherosclerosis in smokers, vapers and nonsmokers 